\title{
Pseudomembranous colitis associated with changes in an ileal conduit
}

\author{
JR SHORTLAND, ${ }^{*}$ RC SPENCER, $\dagger$ JL WILLIAMS $\ddagger$ \\ From the *Departments of Pathology, †Medical Microbiology and $\ddagger$ Urology, Royal Hallamshire Hospital, \\ Sheffield
}

SUMMARY A case of antibiotic associated pseudomembranous colitis following total cystectomy is reported, in which there was involvement of the ileal conduit. The small bowel remaining in situ was uninvolved. Bacteriological studies revealed Clostridium difficile and the toxin in both colon and ileal conduit. Relevant publications concerning pathogenesis are discussed, in relation to the unusual site described in this case. Epidemiological evidence is reviewed which suggests that isolation of patients with pseudomembranous colitis is a logical course of action.

Pseudomembranous colitis is now firmly associated with Clostridium difficile and toxin production. Reported cases often complicate antibiotic therapy particularly ampicillin, and involvement of the colon is the predominant effect, with uncommon and atypical changes in the ileum. We describe a case in which pseudomembranous colitis occurred in a patient following total cystectomy for carcinoma of the bladder. The typical colonic lesions are described along with identical lesions within the isolated ileal loop. The ileum in situ proximal to the ileocaecal valve was uninvolved.

\section{Case report}

A 70-year-old man underwent a total cystectomy in November 1982 for a transitional cell carcinoma of the bladder. He also had ischaemic heart disease and chronic obstructive airways disease. Prior to this the tumour which had been diagnosed in 1979 had been irradiated in 1980 . Initially the lesion appeared to have been controlled but by early 1982 recurrence was detected. Recurrent infections, pain on micturition and intractable bleeding necessitated a cystectomy. During the months prior to cystectomy several courses of antibiotics including cephradine metronidazole and ampicillin, were given for repeated urinary tract infections. Five days after the cystectomy in addition to postoperative metronidazole and neomycin, a course of ampicillin $(500 \mathrm{mg}$ sixhourly for 11 days) was given because of patchy consolidation of the right middle lobe. Twenty-one days after the cystectomy he complained of nausea

Accepted for publication 31 May 1983 and abdominal pain together with vomiting. On examination his abdomen was distended and tense with hyperactive bowel sounds.

Intravenous feeding was instituted until bowel movements which were loose commenced seven days later. $\mathrm{He}$ was allowed home but had to b8 admitted after eight days because of recurrent dia rhoea, abdominal pain and weight loss. His wifs commented that the ileal loop had been offensive for a few days prior to his readmission. He was treated symptomatically for five days with no

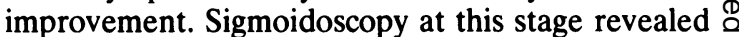
the classical plaques of pseudomembranous colitis. $\overrightarrow{\overrightarrow{0}}$ Despite treatment by vancomycin the patient died 3 the next day, 44 days after his cystectomy and 16 days after the onset of his diarrhoea.

\section{NECROPSY}

The body was that of an elderly man with evidence of considerable recent weight loss. There was severe chronic bronchitis and emphysema. The large bowel revealed the irregular raised yellowish-white plaques set against a hyperaemic mucosa characteristic of pseudomembranous colitis throughout its length (Fig. 1). This change terminated abruptly at the ileocaecal valve. Identical macroscopic features $N$ were present on the mucosa of the ileal conduit $N$ (Figs. 2 and 3). The ureters were intact and drained $N$ into the conduit. Postmortem samples from the $\omega$ rectum, colon and ileal conduit were taken immediately to the bacteriology laboratory. Isolation of $\mathrm{Cl} \stackrel{\circ}{\mathrm{C}}$ difficile was by both a selective medium (Oxoid $\mathrm{Cl} \nsubseteq$ difficile agar base plus 7\% defibrinated horse blood and Oxoid antibiotic supplement) and the use of alcohol, at a final concentration of $50 \%$, to select $\mathbb{D}$ 


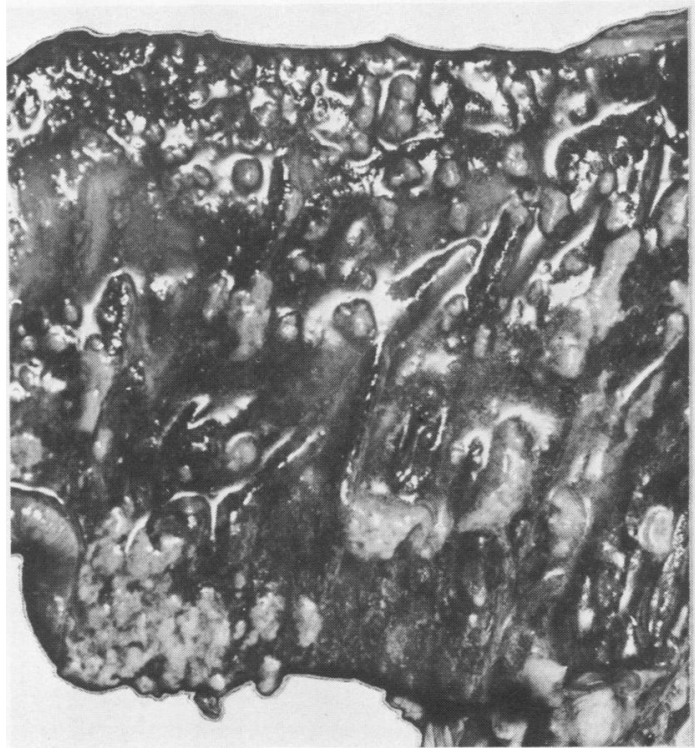

Fig. 1 The colonic mucosa shows aggregates of yellowish-white plaques set against a hyperaemic mucosa throughout its length.

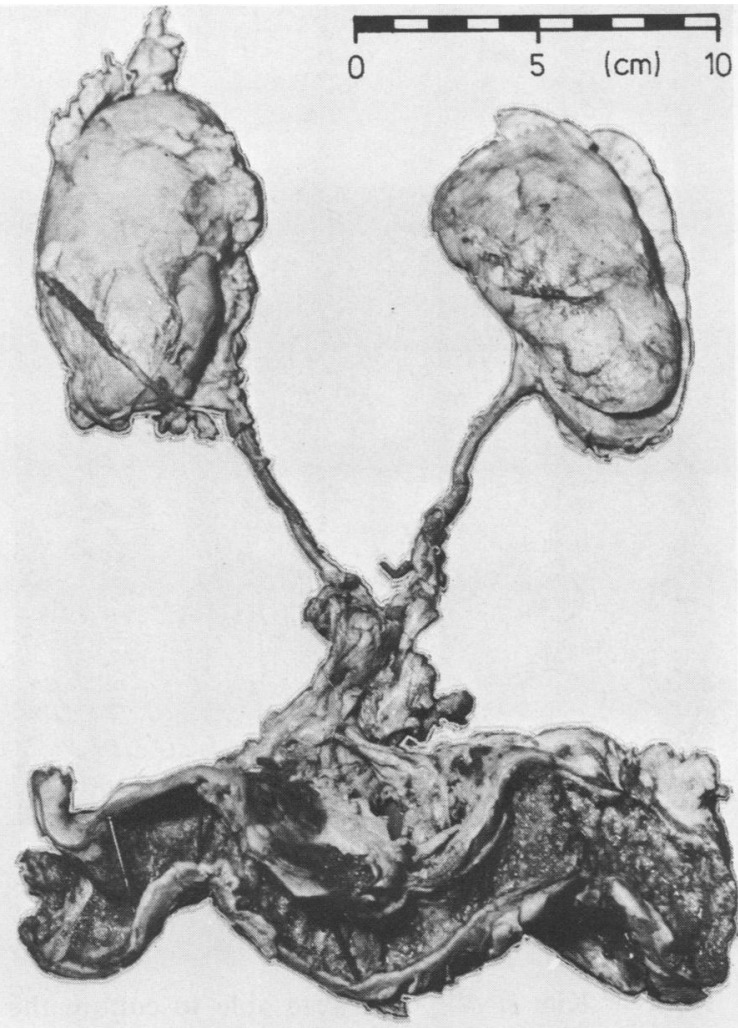

Fig. 2 The ileal conduit in situ with the kidneys and ureters show discrete mucosal plaques. out clostridial spores.' Identification of $\mathrm{Cl}$ difficile isolates and detection of cytotoxin was achieved by standard laboratory methods. ${ }^{2}$ Other gastrointestinal pathogens were sought in the normal way.

\section{HISTOLOGY}

Sections of the large bowel revealed characteristic fibrinous caps overlying distorted glands (Fig. 4). The ileal mucosa revealed autolysis but none of the features of pseudomembranous colitis. In contrast the ileal loop mucosa showed fibrin caps and adjacent mucosal inflammation, all features identical with pseudomembranous colitis (Fig. 5).

\section{BACTERIOLOGY}

Postmortem specimens taken from the rectum colon and ileal conduit revealed $\mathrm{Cl}$ difficile together with cytotoxin. No other pathogens were isolated.

\section{Discussion}

The weight of evidence both from experimental and clinical sources indicates that antibiotic-associated pseudomembranous colitis is of an infective aetiology reviewed by Ashley Price. ${ }^{3} \mathrm{Cl}$ difficile is the organism identified in a number of these cases and the production of a toxin is important in producing experimental lesions. ${ }^{45}$ However, $\mathrm{Cl}$ difficile is a pathogen only rarely found in the intestine of healthy adults. Evidence is emerging that in order for the organism to produce pseudomembranous colitis the bowel must be in a susceptible state. Fekety et $\mathrm{al}^{6}$ found that cultures of $\mathrm{Cl}$ difficile which had been washed free of toxin had no effect on the intestine of normal hamsters; in contrast to the development of caecitis when instilled into animals which had previously been given antibiotics. Pierce et $\mathrm{al}^{7}$ found a predeliction to develop pseudomembranous colitis in patients who had been subjected to diagnostic or therapeutic gastrointestinal procedures, and related this to possible bowel stasis. Thus it appears that in order to contract pseudomembranous colitis a patient must have a susceptible intestine and come into contact with the organism. Credence is given to this concept since animals survive when kept in sterile or non-contaminated conditions after vancomycin treatment ${ }^{6}$ or clindamycin administration, ${ }^{8}$ but contract pseudomembranous colitis if a contaminated animal is introduced. ${ }^{8}$ Environmental factors are relevant clinically. Miller and Jick ${ }^{9}$ pointed out clustering of cases and this has been subsequently substantiated ${ }^{7}$ all adding to the concept of an infective lesion. Burdon et al ${ }^{10}$ report the introduction of the disease into a ward with the transfer of a patient with pseudomembranous colitis. Recently Malamou-Ladas et $a^{11}$ examined the en- 


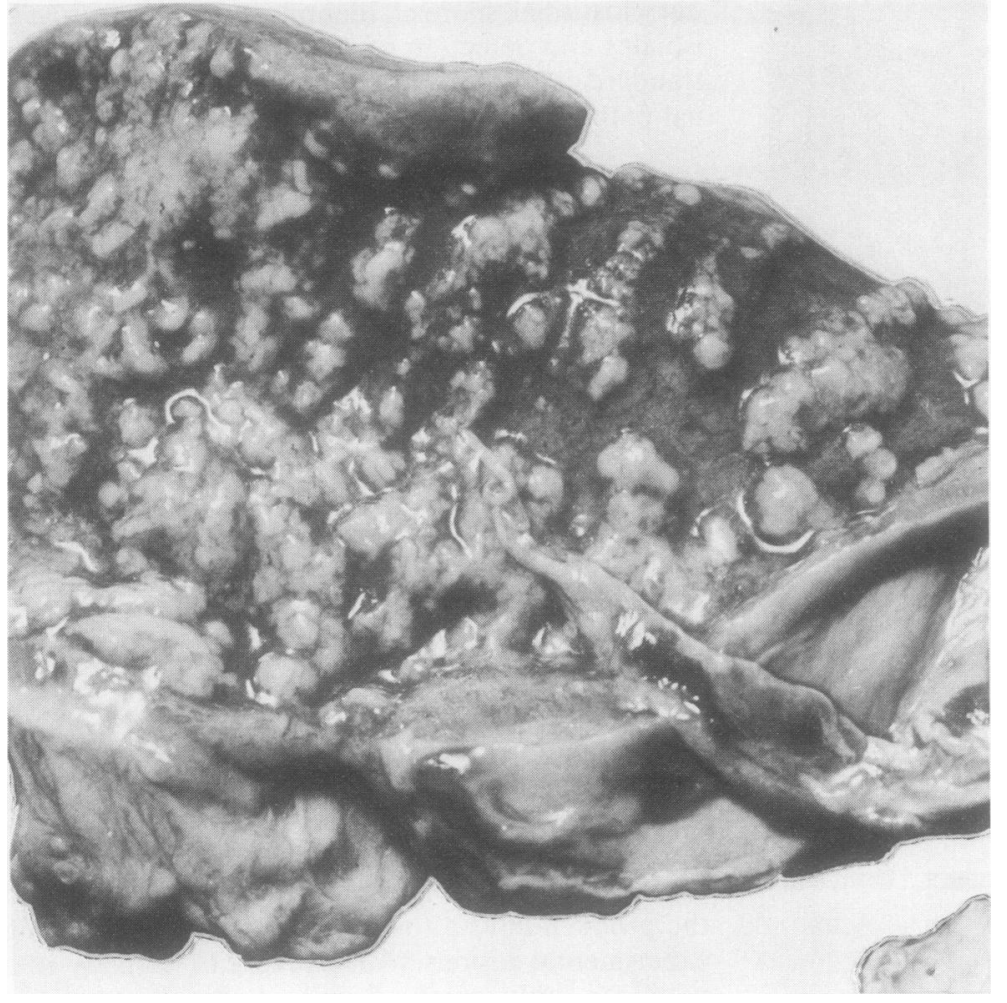

Fig. 3 Mucosal plaques against a hyperaemic background in the ileal conduit.

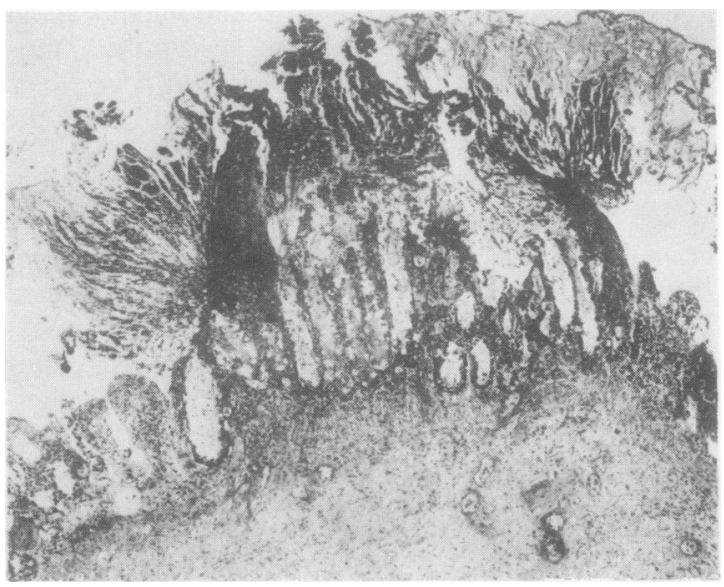

Fig. 4 A fibrin cap infiltrated by polymorphs overlying dilated colonic mucosal glands characteristic of pseudomembranous colitis. The adjacent mucosa is autolysed but mildly inflamed. Haematoxylin and eosin $\times$ 35.

vironment of hospital wards and isolated the organism in significant numbers from items subject to faecal contamination. Their work substantiates the

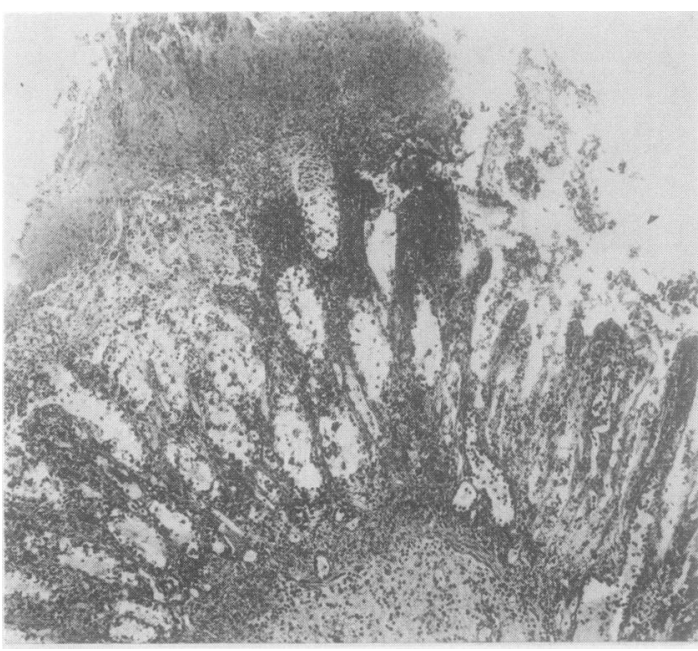

Fig. 5 Changes identical with pseudomembranous colitis are seen in the small intestinal mucosa of the ileal conduit. Haematoxylin and eosin $\times 55$.

work of Kim et al ${ }^{12}$ who were able to culture the 0 organism not only from inanimate objects but from 0 the hands and stools of asymptomatic hospital per- 
sonnel. Household pets may act as a reservoir, and this may be increased by antibiotic therapy. ${ }^{13}$

Whilst ampicillin was the antibiotic used in our case Bartlett ${ }^{14}$ reports pseudomembranous colitis associated with a wide range of antimicrobial agents and points out that nearly any antibiotic may be responsible. Cases of pseudomembranous colitis associated with antibiotics usually affect the large bowel only and stop abruptly at the ileocaecal valve. This was the situation in the case reported, the terminal ileum showed no evidence of plaques. The only part of the small intestine involved in our case was the segment isolated as the ileal conduit draining the ureters. The pattern of involvement was identical to that seen in the colon and contrasted with the less discrete circumferential pattern seen when pseudomembranous colitis traverses the ileocaecal valve. The local conditions in the ileal loop must have rendered the mucosa more susceptible than that in the remainder of the small intestine. The cause of the increased susceptibility remains something of an enigma. An endogenous bowel metabolite such as bile acids ${ }^{15}$ would appear untenable in this case since the loop had been totally isolated from the bowel for some six weeks. The physicochemical milieu of the intestinal mucosa is considerably modified in a loop. This may play an important role in increasing adherence and penetration of organisms as suggested by Smith ${ }^{16}$ thus allowing the clostridium to colonise the conduit in contrast to the in situ small intestine.

The presence of microthrombi in the lesions suggests the possibility of a local Shwartzman reaction. This has been investigated by Behan and Mills ${ }^{17}$ who demonstrated reduced serum complement concentrations in patients with pseudomembranous colitis and make the analogy to endotoxic shock. The numbers of cases are small and the complement profiles are not strictly comparable. The concentrations quoted could equally be interpreted as a consequence of pseudomembranous colitis rather than the cause. The evidence for a local Shwartzman reaction in pseudomembranous colitis remains equivocal.

The indications are that pseudomembranous colitis is an infective disease. Potential sources of infection include not only inanimate objects subject to faecal contamination, but the hands of hospital personnel in contact with affected patients and objects contaminated by them. Organisms can survive on a floor for up to five months ${ }^{12}$ and isolation of such patients would appear to be logical.
We wish to thank Mr M Eaton for the photographic work and Mrs M Hogg for typing the manuscript.

\section{References}

${ }^{1}$ Borriello SP, Honour P. Simplified procedure for the routine isolation of Clostridium difficile from faeces. J Clin Pathol 1981;34:1124-7.

${ }^{2}$ Burden DW. Laboratory investigation of antibiotic-associated diarrhoea. ACP Broadsheet 102, 1982.

${ }^{3}$ Price AB. Pseudomembranous colitis. In: Wright R, ed. Recent advances in gastrointestinal pathology. London: W B Saunders Co Ltd 1980:151-72.

${ }^{4}$ Rifkin GD, Silva J, Fekety R. Gastrointestinal and systematic toxicity of fecal extracts from hamsters with clindamycininduced colitis. Gastroenterology 1978;74:52-7.

${ }^{5}$ Larson HE, Price AB, Honour P, Borriello SP. Clostridium difficile and the aetiology of pseudomembranous colitis. Lancet 1978; ;:1063-6.

' Fekety R, Silva J, Toshniwal R, et al. Antibiotic associated colitis: effects of antibiotics on Clostridium difficile and the disease in hamsters. Rev Infect Dis 1979;i:386-97.

' Pierce PF, Wilson R, Silva J, et al. Antibiotic-associated pseudomembranous colitis: an epidemiologic investigation of a cluster of cases. J Infect Dis 1982;145:269-74.

${ }^{8}$ Larson HE, Price AB, Borriello SP. The possible role of environmental Clostrium difficile in antibiotic colitis of hamsters and man. Clin Res 1979;27:349A.

9 Miller RR, Jick H. Antibiotic-associated colitis. Clin Pharmacol Ther 1977;22:1-6.

${ }^{10}$ Burdon DW, Mogg GAG, Alexander-Williams J, et al. Epidemiology of antibiotic-associated colitis. 11th International Congress of Chemotherapy and 19th Interscience Conference on Antimicrobial agents and Chemotherapy. Abs No 839, 1979.

' Malamou-Ladas H, O'Farrell S, Nash JQ, Tabaqcha LS. Isolation of Clostridium difficile from patients and the environment of hospital wards. J Clin Pathol 1983;36:88-92.

${ }^{12} \mathrm{Kim} \mathrm{KH}$, Fekety R, Batts DH, et al. Isolation of Clostridium difficile from the environment and contacts of patients with antibiotic-associated colitis. J Infect Dis 1981;143:42-50.

${ }^{13}$ Borriello SP, Honour P, Turner T, Barclay F. Household pets as a potential reservoir for Clostridium difficile infection. J Clin Pathol 1983;36:84-7.

14 Bartlett JG. Antimicrobial agents implicated in Clostridium difficile toxin-associated diarrhoea or colitis. Johns Hopkins Med J 1981;149:6-9.

15 Hofman AF. Bile acids, diarrhoea and antibiotics: data, speculation and a unifying hypothesis. J Infect Dis 1977;135:S126S132.

${ }^{16}$ Smith $\mathrm{H}$. Microbial surfaces in relation to pathogenicity. Bacteriol Rev 1977;41:475-500.

17 Behan WMH, Mills PR. Possible evidence for a Shwartzman reaction in pseudomembranous colitis. Digestion 1982; 23:141-50.

Requests for reprints to: Dr JR Shortland, Department of Pathology, University of Sheffield Medical School, Beech Hill Road, Sheffield S10 2RX, England. 